Meta

Journal des traducteurs

Translators' Journal

\title{
De la traduction (juridique) à la jurilinguistique. Fonctions proactives du traductologue
}

\section{Jean-Claude Gémar}

Volume 50, numéro 4, décembre 2005

Pour une traductologie proactive - Actes

For a Proactive Translatology - Proceedings

Por una traductología proactiva - Actas

URI : https://id.erudit.org/iderudit/019840ar

DOI : https://doi.org/10.7202/019840ar

Aller au sommaire du numéro

Éditeur(s)

Les Presses de l'Université de Montréal

ISSN

0026-0452 (imprimé)

1492-1421 (numérique)

Découvrir la revue

Citer cet article

Gémar, J.-C. (2005). De la traduction (juridique) à la jurilinguistique. Fonctions proactives du traductologue. Meta, 50(4). https://doi.org/10.7202/019840ar
Résumé de l'article

Plus ancienne que ce que l'on pense, la jurilinguistique, révélée par la traduction, s'est développée comme discipline dans le dernier tiers du $\mathrm{XX}^{\mathrm{e}}$ siècle. Le Canada, pour des raisons tenant à son histoire, ses langues et ses traditions juridiques, a joué un rôle de pionnier dans cette évolution. Répandue dans le monde entier, la jurilinguistique n'est pas le monopole des régimes de common law. Née sous la pression des besoins de communication et de diffusion de l'Etat moderne, puis de la société civile, elle transcende les frontières des langues et les barrières des traditions juridiques. Un corps de doctrine se constitue progressivement et le savoir-faire des jurilinguistes s'exporte. Ils contribuent à édifier un savoir d'un genre nouveau caractérisant une discipline à la croisée du droit et de la linguistique, aux fonctions liées à une pratique, à sa transmission et à son évolution.
Ce document est protégé par la loi sur le droit d'auteur. L'utilisation des services d’Érudit (y compris la reproduction) est assujettie à sa politique d'utilisation que vous pouvez consulter en ligne.

https://apropos.erudit.org/fr/usagers/politique-dutilisation/ 


\title{
De la traduction (juridique) à la jurilinguistique. Fonctions proactives du traductologue
}

\author{
JEAN-ClAUDE GEMAR \\ ETI, Université de Genève, Genève, Suisse \\ Jean-Claude.Gemar@eti.unige.ch
}

« [I]1 est toujours possible de dire la même chose autrement »

Paul Ricoeur (2004 : 45)

\begin{abstract}
RESUME
Plus ancienne que ce que l'on pense, la jurilinguistique, révélée par la traduction, s'est développée comme discipline dans le dernier tiers du $\mathrm{XX}^{\mathrm{e}}$ siècle. Le Canada, pour des raisons tenant à son histoire, ses langues et ses traditions juridiques, a joué un rôle de pionnier dans cette évolution. Répandue dans le monde entier, la jurilinguistique n'est pas le monopole des régimes de common law. Née sous la pression des besoins de communication et de diffusion de l'Etat moderne, puis de la société civile, elle transcende les frontières des langues et les barrières des traditions juridiques. Un corps de doctrine se constitue progressivement et le savoir-faire des jurilinguistes s'exporte. Ils contribuent à édifier un savoir d'un genre nouveau caractérisant une discipline à la croisée du droit et de la linguistique, aux fonctions liées à une pratique, à sa transmission et à son évolution.
\end{abstract}

\begin{abstract}
While it has only taken shape as a discipline in the last quarter of the twentieth century and in the wake of translation, jurilinguistics is in fact a practice far older than generally understood. Canada has, for reasons related to its history, languages and legal traditions, acted as a pioneer in the development of jurilinguistics as it has spread across the world, well beyond common law countries. Precipitated by the requirements relating to the communication of ideas, both in the modern state and civil society generally, jurilinguistics as a field of endeavour transcends linguistic barriers and legal traditions. Through their sharing of experiences and skills, jurilinguists have generated a body of research at the crossroads of law and linguistics. Their role has been central to the practice, communication and development of this new field of scholarship.
\end{abstract}

\section{MOTS-CLÉS/KEYWORDS}

jurilinguistique, traductologie, langues de spécialité, droit, formation

J'ai montré ailleurs (Gémar, 1982, 1994) comment la Nouvelle-France, une fois passée aux mains des Anglais, au XVIII ${ }^{\mathrm{e}}$ siècle, puis le Canada, au $\mathrm{XIX}^{\mathrm{e}}$ siècle et dans la première moitié du $\mathrm{XX}^{\mathrm{e}}$, avaient longtemps laissé saccager la langue française dans les traductions de leurs textes officiels. Puis comment, par un de ces revirements pendulaires que connaît l'Histoire, l'État canadien et les pouvoirs publics québécois avaient pris sa défense avant de l'illustrer dans la seconde moitié du $\mathrm{XX}^{\mathrm{e}}$ siècle.

Et cela grâce à la traduction - la juridique particulièrement - et à ses praticiens, avant qu'elle ne se développe, au cours des dernières décennies, en traductologie. Cette jeune science, encore en formation, ne cesse de se renouveler, de se transformer en prenant appui sur des disciplines complémentaires ou connexes, telles que l'informatique et la psychologie et, bien sûr, la linguistique, notamment appliquée. Celle-ci a donné naissance à de nombreux avatars, dont la linguistique du texte, l'analyse du discours et la terminologie. Le découpage de la langue en « langues de spécialité » ou «spécialisées » (Lerat 1995) selon une vision pratique des applications d'une langue à un domaine de l'activité humaine (médecine, droit, commerce, transports, etc.) s'est étendu à l'étude des langages : du droit, de la médecine, de la bourse ou... du transport maritime, créant autant de spécialités dont se sont saisis les traducteurs. Ainsi est né l'intérêt des traducteurs, 
puis de la traductologie, pour la jurilinguistique ${ }^{1}$, qui, contrairement à ce que $1^{1}$ 'on croit généralement, ne date pas d'hier. Elle remonte à la préhistoire du droit et de ses écrits, aux premiers tâtonnements des rédacteurs de codes des droits cunéiformes, dont le Code de Hammurabi (1694 av. J.-C) reste le monumental exemple. Les premiers jurilinguistes étaient des praticiens, car l'écriture du droit, comme tout acte d'écriture, est savoir-faire humain avant d'être pratique professionnelle, ce qui, en cela, ne diffère guère de celle de l'écrivain public d'antan. Elle a néanmoins donné rapidement naissance (cf. Justinien et son œuvre) à des considérations et à des réflexions préfigurant les théorisations à venir, inspirées des pères fondateurs de la pensée occidentale que furent les Platon, Aristote et autres grands esprits essentiels. La justice, les lois et les règles en découlant ne laissaient pas ces derniers indifférents, et la forme de leur production les préoccupait parfois au plus haut point. Les exemples abondent, de Cicéron à Blackstone jusqu'à un Lord Denning, le célèbre juge anglais, donnant ce conseil : «Vous devez utiliser des mots simples et clairs que tous vos auditeurs et lecteurs puissent comprendre» (Malaurie, $1996: 278$ ).

\section{Du langage du droit à la jurilinguistique}

Depuis toujours, la manière de rédiger les lois et les autres textes porteurs de règles juridiques ne cesse de préoccuper le monde des juristes, praticiens et théoriciens confondus. En effet, si « nul n'est censé ignorer la loi » [du latin Nemo censetur legem ignorare], comment la faire connaitre de la meilleure façon à ceux-là mêmes à qui elle est destinée ? Chaque peuple, selon sa culture, ses us et coutumes, a forgé sa propre tradition de rédaction des textes juridiques. Avec bonheur parfois, lorsque l'accent est mis sur la clarté, la concision et la précision du message. Mais, le plus souvent, lorsque dans le même texte sont conjugués lourdeur, maladresse et verbiage, c'est au détriment du citoyen, de l'usager que s'exprime le droit. Chaque langue, chaque culture juridique en porte le témoignage ironique, sous la plume satirique de ses grands écrivains, les Chaucer, Rabelais, Shakespeare, Molière, Swift ou Voltaire, pour ne parler que des langues de Flaubert et de Faulkner. Le langage du droit fascine le profane autant qu'il le rebute, sans doute parce que, comme le pense Rouland (1991 : 14), «l'exigence de formes et l'hermétisme du langage [du droit] nous découragent autant qu'ils nous contraignent ». Le langage humain recèle toujours une grande part de mystère s'apparentant au sacré : Au commencement était le Verbe ${ }^{2}$. A quoi répond, en lointain écho: «Au commencement était la Règle. », le cri de Carbonnier (1995: 96). Plus qu'aucun autre domaine, hormis le religieux, le langage du droit incarne le mythe prométhéen de la naissance et de la diffusion d'un savoir, celui dont le juriste serait investi, où le mythique le disputerait au mystique (Nida, $1982: 261$ ).

Ce langage est au cœur des préoccupations des juristes, qui nous rappellent régulièrement que le droit est affaire de mots. Et cela dans tout l'univers du droit, vers quelque côté que l'on se tourne, dans le monde juridique anglophone - où, pour Mellinkoff (1963 : vii), " The law is a profession of words » - comme dans l'espace francophone - où, d'après Gény (1921:456) «prise dans son ensemble, la technique juridique aboutit, pour la plus grande part, à une question de terminologie ». Chacun pourra le vérifier par la consultation d'ouvrages parmi les plus récents et les plus fouillés produits par la doctrine, tels que le Dictionnaire de droit privé du Québec (CRDPCQ, 1985) et, en France, le Vocabulaire juridique (Cornu, 1987) et le Dictionnaire de la culture juridique (Alland, 2003). On remarquera, dans ce dernier ouvrage notamment, la place qu'occupe l'entrée «Linguistique juridique » (p. 952), rédigée par Cornu. Ce terme consacre, sous une étiquette dorénavant des plus classiques, l'apport indiscutable à la science juridique d'une discipline, la linguistique, qui, un quart de siècle plus tôt, laissait sceptiques certains des plus éminents linguistes de l'époque devant l'intérêt pratique qu'elle pouvait présenter pour les juristes (Mounin, 1979 : 9). Or, depuis, la linguistique, théorique autant qu'appliquée, s'est révélée des plus utiles pour les juristes, quelles que soient leurs réticences devant ce « droit d'ingérence » des linguistes (au sens large du terme) dans leur discipline. Ils en ressentirent pourtant le besoin lorsqu'il fallut se lancer dans des entreprises, nouvelles pour eux, telles que la création de banques de terminologie (juridique), la lexicographie (juridique) (unilingue, bi- et multilingue), la réforme du vocabulaire, des textes et des formulaires administratifs (en cours en France, par ex.), la corédaction législative et la révision des lois et d'autres textes juridiques (le contrat, par ex.), pour ne rien dire 
de l'interprétation des lois (Côté 1999) grâce, en partie, aux avancées des sciences linguistiques. Toute science bénéficie des progrès accomplis dans les champs avoisinants, souvent complémentaires. La solution d'un problème ne pouvant être trouvée à l'intérieur des seules frontières d'une discipline, l'apport d'autres sciences est, nous dit Leroi-Gourhan (1983 : 599), nécessaire parce que «le biologiste sait bien que sa science repose sur la physique, un physicien sait bien qu'il ne peut rien sans les mathématiques, etc. ». Le droit ne fait pas exception à la règle et la linguistique lui sert de discipline d'appoint lorsqu'il s'agira de se lancer dans l'écriture du droit, cet « exercice impitoyable pour le juriste » (Badinter 1991). La jurilinguistique se situe à la croisée des deux disciplines mères que sont pour elle la linguistique et le droit.

C'est ainsi que les mots du droit constituent, dans le sens spécialisé que leur donnent les juristes, la langue (ou terminologie) juridique, elle-même sous-ensemble de la langue générale, mais apparentée à un langage technique ${ }^{3}$. Mise en discours (Darbelnet $1982: 51$ ), avec le concours des mots de la langue courante, cette langue produit le langage du droit ${ }^{4}$. Ce langage varie d'une langue et d'une culture juridique à l'autre, parfois par l'entremise d'un système différent du nôtre. C'est ici que la jurilinguistique présente son utilité, voire sa nécessité, par les fonctions que le jurilinguiste peut être amené à remplir selon la situation linguistique et juridique dans laquelle il exercera ses talents. En commençant par les fonctions, je me réfère encore une fois aux fonctions cardinales faire, transmettre, chercher - que l'anthropologie a dégagées de longue date (Dumézil 1995) et que la traductologie a reprises à son compte (Ladmiral 1988:36).

De toute évidence, la difficulté croît à proportion du nombre de langues et de systèmes en cause, ce qui ne simplifiera pas la tâche du jurilinguiste, quelle que soit la fonction qu'il assumera. La première est celle de l'activité qu'il pratique et qui lui permet d'exercer son savoir-faire, ce par quoi je commencerai. La deuxième consiste justement à transmettre ce savoir-faire par la formation, l'exemple, à l'image des « compagnons » d'autrefois inculquant le goût du travail bien fait à leurs apprentis. La troisième est indispensable à la vie, au développement et au renouvellement de toute discipline quelle que soit sa vocation, littéraire ou scientifique. C'est celle de la recherche qui, lorsqu'elle est conduite avec la rigueur et l'esprit scientifique nécessaires, insuffle par ses applications le désir de dépassement générateur de progrès et d'innovation.

\section{La jurilinguistique : une pratique}

La jurilinguistique est avant tout un savoir-faire personnel qui a évolué en pratique professionnelle. Cette fonction « productive » de la jurilinguistique est sans doute la plus évidente qui, à l'instar de la traduction, s'exerce depuis que l'on rédige, réforme, compose, traduit, révise, bref, produit des textes reconnus comme étant juridiques parce qu'ils visent à créer ou à réaliser du droit (Cornu, 2000 : 21). Cette pratique est omniprésente dans toutes les grandes traditions et langues juridiques. On trouve de nombreuses références sur le sujet, notamment sur la production franco-canadienne (Doiron \& Snow, 2005 : ????), comme en attestent en particulier les copieuses bibliographies de thèses de doctorat soutenues en traduction juridique au cours des dernières années, au Canada, en Espagne et en France, soutenances auxquelles - à titre de directeur, codirecteur ou de juré - il m'a été donné d'assister. On aurait donc tort de penser que les problèmes engendrés par des langues (juridiques) en contact se limitent au seul Canada ${ }^{5}$. Nul n'est épargné, pays unilingues y compris ${ }^{6}$. Mais le problème ne se pose pas seulement à propos des langues en contact, c'est le langage du droit lui-même qui est à la source des difficultés que dénoncent certains auteurs ${ }^{7}$.

Le rythme des activités en la matière, parallèlement aux besoins croissants de la société, s'est accéléré au cours du dernier quart de siècle, et de nombreux groupes de jurilinguistique ont vu le jour. Au Canada notamment, tant dans le domaine public, auprès des différents gouvernements, offices, commissions et conseils - soit l'Administration en général - que dans la sphère privée : associations de juristes, barreaux, universités, entreprises, études et cabinets (d'avocats, de juristes et de traducteurs ${ }^{8}$ ), etc. Toutefois, ce sont encore ces derniers qui, assistés des terminologues, en pratiquant parfois intensivement la jurilinguistique à des fins purement professionnelles, lui ont donné ses lettres de créance, comme le démontre, entre autres, l'histoire du Canada, où la traduction a tenu un rôle peu commun 9 . 
L'intérêt des juristes pour ces questions ne faiblissant $\operatorname{pas}^{10}$, la jurilinguistique se développe, et cela à l'échelle du monde, parallèlement aux difficultés croissantes que génèrent, à l'ère planétaire et sous la pression des besoins de la société, la communication contemporaine (Morin 1999) et ses nombreux modes d'expression. Toutefois, les difficultés qu'engendre, urbi et orbi, le commerce du droit et de la langue n'en demeurent pas moins. Le constat est édifiant à cet égard si l'on prend le cas de l'Union européenne comme exemple limite de ce que la confrontation des langues et du droit peut produire de difficultés de toute sorte (Gozzi 2001 : 23). Les chiffres sont accablants. Selon J.-F Burgelin, la Cour de Cassation « est saisie de 30000 pourvois par an et rend 27000 arrêts ${ }^{11}$. Norbert Rouland (1991 : 27) parle, quant à lui, de l'année 1978 qui « vit la prolifération de 1250 lois et de 1308 décrets ». On a fait mieux depuis. Les Etats-Unis, à eux seuls, génèrent chaque année quelque 300000 pages de textes réglementaires. Le Congrès des É.-U., en 2001, a traité 5500 projets de loi $^{12}$. L' « acquis communautaire » de 1'Union européenne

représente, selon Tito Gallas (2002 : 97) quelque 85000 pages - que doit d'ailleurs traduire chacun des pays candidats à l'entrée dans l'Union -, ce qui, dans les 11 langues officielles actuelles, donne «258 221 documents du Conseil ». Triste bilan de la prolifération des textes juridiques que produisent les Etats modernes et organisations. À cette aune, on mesurera, à grand peine et non sans une certaine angoisse, le volume vertigineux de la production du secteur privé. Comment, dans de telles conditions, produire à la fois la quantité et la qualité, de surcroît dans des délais que l'on souhaite raisonnables ? Tel est le dilemme de la jurilinguistique, que les jurilinguistes de tous bords s'efforcent de résoudre au quotidien. Cette quête du Graal, ils la poursuivent le plus souvent avec bonheur et quelquefois avec la frustration de ne pas avoir atteint le but désiré. Et cela pour la simple raison qu'écrire est quelque chose qu' on ne peut jamais faire aussi bien que cela pourrait être fait (Hemingway).

La présence matérielle de la jurilinguistique et les effets d'une activité en plein développement se donnent désormais à voir. Ce terme apparaitt, en français comme en anglais (jurilinguistics) dans TERMIUM, l'une des grandes banques mondiales de terminologie, et diverses publications, canadiennes notamment. L'activité jurilinguistique et les besoins qu'elle met en évidence n'ont pas manqué de faire réfléchir les pouvoirs publics sur les meilleures façons de communiquer par écrit avec le public. Des portails et des sites se trouvent à la disposition de tous, où les intéressés professionnels, sociétés, particuliers, profanes ou amateurs - peuvent aller voir comment résoudre telle difficulté textuelle ou linguistique ou rédiger, réviser, réécrire, voire traduire tel document ${ }^{13}$. Il n'est pas jusqu'à la dictionnairique, activité langagière hautement spécialisée s'il en est, qui n'ait retenu l'intérêt d'un groupe de terminologues/graphes (ou de lexicologues/graphes), et même de groupes de juristes ${ }^{14}$.

Se pose alors la question de la transmission de ce savoir-faire, voire du savoir constitué par la pratique de la jurilinguistique.

\section{Transmettre le savoir(-faire)}

Parce qu'elle s'effectue sur les lieux de travail, la transmission du savoir-faire est surtout le fait de praticiens. Les théoriciens n'y sont cependant pas étrangers, car leurs travaux, réflexions et modèles théoriques influencent la pratique. Ce que nous verrons dans la partie suivante, la dernière, consacrée à la jurilinguistique théorique. Disons ici, à titre d'illustration, que la production de dictionnaires, lexiques et autres vocabulaires unilingues, bi- et multilingues, est étroitement liée aux progrès accomplis en lexicologie, dont la lexicographie, générale ou spécialisée, est une application. Les centres ${ }^{15}$, instituts ${ }^{16}$ et groupes de recherche ${ }^{17}$ (en droit, droit comparé, linguistique, traduction, terminologie, etc.) où l'on produit une certaine forme de jurilinguistique, contribuent, outre à la transmission d'un savoir, également à inculquer un savoir-faire.

C'est toutefois dans les universités, en amont, que se prépare cette transmission. C'est là, en effet, que par l'enseignement du droit, de la linguistique, de la traduction, de la terminologie, etc., sont jetées les bases des éléments et données qui permettront d'édifier et de développer la jurilinguistique. Mais pour cela, il faudra d'abord que ces disciplines, par l'effet du hasard, d'un intérêt particulier ou par simple nécessité viennent à se croiser (Greenstein 2003) pour, après avoir 
franchi l'obstacle des préjugés disciplinaires, parvenir à se compléter et, le cas échéant, faire cause commune $^{18}$.

Cela dit, le rôle que jouent les gouvernements et leurs administrations, les organisations internationales (ONU) et régionales (UE) dans la formation à la jurilinguistique et sa transmission est primordial ${ }^{19}$. On peut avancer que, sur ce plan et en matière législative tout particulièrement laquelle est au centre de l'action des jurilinguistes -, l'Administration fédérale du Canada et notamment son ministère de la Justice ${ }^{20}$, les gouvernements du Québec puis du NouveauBrunswick, entre autres, et leurs administrations ont fait œuvre pionnière (Gémar 1983). Les pouvoirs publics, à l'échelle fédérale comme dans les provinces, ont en outre encouragé et soutenu nombre d'initiatives et de projets de formation et de perfectionnement présentés par des associations professionnelles, des groupes d'intérêt privé ou public. La protection du consommateur contre les abus de toute sorte - linguistiques y compris - en est un bon exemple. Au Québec, la Loi sur la protection du consommateur ${ }^{21}$ prévoit en effet, à l'article 25, qu'un contrat relatif aux biens et aux services « doit être clairement et lisiblement rédigé ». L'intention du législateur, en l'occurrence, est clairement jurilinguistique. L'action de groupes et d'associations pour l'illustration de la langue, voire sa défense, est aussi à signaler parce qu'ils oeuvrent pour l'éducation du public ${ }^{22}$.

L'action combinée de ces institutions, organismes et groupes divers contribue à transmettre et à développer le savoir-faire jurilinguistique de par le monde ${ }^{23}$. Un savoir en découle qui puise ses racines dans les travaux, recherches et réflexions des linguistes, juristes, traducteurs, terminologues et autres langagiers. Car que seraient, à quoi serviraient une pratique qui ne serait pas suivie d'une réflexion critique et, à l'inverse, une théorie qui tournerait sur elle-même, à vide, sans prise sur un réel que « prises isolément, ni la pratique ni la théorie ne suffisent à construire » (Rouland1991 : $15)$ ?

\section{Penser la jurilinguistique ou la fonction théorique}

Encore à l'état embryonnaire quand on la compare à d'autres disciplines comme le droit ou la linguistique, dont le statut de science est établi et reconnu, la réflexion théorique due aux jurilinguistes n'est pas négligeable pour autant.

Réfléchir sur une pratique afin de mieux la comprendre et, peut-être, de l'améliorer, de la perfectionner est le propre de l'homme. Cela n'aboutira pas nécessairement à une réflexion théorisante, mais peut la favoriser. Même si les difficultés inhérentes à une démarche susceptible de déboucher sur des réflexions d'ordre philosophique, voire épistémologique, sont de nature à décourager l'apprenti théoricien, la jurilinguistique fait partie des activités humaines offrant prise à une certaine forme de théorisation, donc de recherche. Je parle ici de recherche " appliquée », la recherche dite « fondamentale » étant, en l'espèce et en l'état actuel des choses, pure utopie. En outre, la nature interdisciplinaire de la jurilinguistique ne pouvait manquer de retenir l'attention de chercheurs venus d'autres horizons et disciplines, favorisant ainsi la naissance d'une forme de discours «metajurilinguistique », où interviennent apports et démarches extérieurs au droit mais fondés sur un intérêt commun pour son langage (Gémar \& Kasirer 2005).

Toutefois, en ce début du XXI ${ }^{\mathrm{e}}$ siècle, parler de théorie en jurilinguistique serait exagéré. Par rapport aux sciences juridiques ou à la psychologie et à leurs savants et théoriciens, cela équivaudrait à comparer la machine à calculer de Pascal et un ordinateur. Un monde de progrès scientifiques et technologiques les sépare. On ne saurait pas davantage comparer les réflexions d'un légiste anonyme du XVIII siècle et les prémisses d'une solide réflexion générale sur le langage du droit que sont The Language of the Law (Mellinkoff 1963) et Le langage du droit (Sourioux et Lerat 1975). La différence des démarches entre ces deux ouvrages apparaît clairement, la présence d'un linguiste (Lerat) complétant harmonieusement le rôle du juriste (Sourioux). Le progrès accompli tient au supplément de savoir injecté dans la réflexion scientifique univoque de départ.

Néanmoins, l'activité des jurilinguistes n'a guère pâti de cette absence de fondements théoriques durant les quelques décennies de la seconde moitié du $\mathrm{XX}^{\mathrm{e}}$ siècle où elle s'est exercée et au fil desquels elle s'est essentiellement nourrie de sa propre pratique. Depuis, les ouvrages, travaux 
et recherches consacrés au sujet, dans les différentes branches et disciplines composant la jurilinguistique, se sont succédé à un rythme qui va s'accélérant et constituent déjà un corpus de doctrine bien établi ${ }^{24}$. Toutefois, comme la multiplication des points de vue et la diversité des approches n'induisent pas nécessairement un approfondissement de la réflexion, aucune discipline ne semble s'être imposée par devers les autres. Aussi la linguistique, avec ses multiples courants tributaires, demeure-t-elle la voie d'accès principale pour les chercheurs travaillant sur la langue, le texte et les corpus, dont les juridiques (Chodkiewicz 2005 :23). On le constatera dans les exemples donnés plus haut (cf. note précédente), bien que, selon les sujets, les thèmes et textes abordés, telle discipline puisse être invoquée de préférence à telle autre. Rien n'empêche d'envisager la question du point de vue d'un sociologue, d'un historien, d'un philosophe ou d'un psychologue, pour ne prendre que ces disciplines comme exemples.

En somme, le jurilinguiste praticien et son homologue théoricien ne peuvent se passer l'un de l'autre. Ils sont parfois réunis dans la même personne, tels le juge Pigeon au Canada, le doyen Cornu en France et Brian Garner ${ }^{25}$ aux Etats-Unis. Ces grands praticiens ont fait œuvre originale à partir d'une réflexion personnelle inspirée par la confrontation quotidienne avec les difficultés que pose le texte juridique proprement dit : loi, jugement, acte, et parfois même celui de doctrine - tel un dictionnaire. Ce faisant, ils ont notablement contribué à fonder la discipline « inductive » (Ladmiral 1988) que pourrait devenir la jurilinguistique au contact et à l'exemple de la traductologie.

\section{Conclusion}

Pour réaliser le texte juridique, les nombreuses méthodes, théories, formules et autres manières de voir ou d'aborder la rédaction d'un texte destiné à la communication ne doivent pas être écartées a priori. Chacune a sa justification à une étape quelconque de la production du texte, depuis sa conception jusqu'à son application et même son interprétation par les tribunaux. Dans cette entreprise, désormais, le rôle du jurilinguiste n'est plus superflu. Comme la terminologie pour la traduction, la jurilinguistique peut accompagner le droit, lui servir de « science auxiliaire » (Mounin 1979 : 9), particulièrement dans les situations où celui-ci est confronté à des textes devant l'exprimer dans une autre langue, et a fortiori dans plusieurs autres.

Si le but de la connaissance, comme le pense Edgar Morin (1984: 292), « est de dialoguer avec le mystère du monde », la jurilinguistique peut contribuer à lever un coin du voile recouvrant le langage du droit et ses textes. Dans cette entreprise, l'exemple du Canada pourrait illustrer le conseil du Montesquieu de L'Esprit des lois suggérant que les lois éclairent l'histoire d'un pays, et réciproquement.

\section{NOTES}

1. Qui consiste à appliquer un traitement linguistique aux textes juridiques sous toutes leurs formes. La jurilinguistique ne doit pas être confondue avec l'étude du droit du langage, des langues ou des droits linguistiques, (cf. TERMIUM, OBS, sous l'entrée « jurilinguistics »), puisqu'il s'agit alors d'étudier ou d'analyser le droit ou un droit, et non son mode d'expression: le langage. Quant au terme « jurilinguistique », que dire d'autre, en effet, quand on analyse sa formation et la compare à d'autres solutions? Qu'elle est conforme à l'esprit comme aux règles de la morphologie française, car jurilinguistique est construit sur le mot-suffixe «linguistique» qui a donné naissance, dans le domaine des sciences linguistiques, à une famille nombreuse de termes exprimant le fond des préoccupations du linguiste et l'orientation de sa recherche : sociolinguistique, psycholinguistique, neurolinguistique, ethnolinguistique et, plus récemment, etholinguistique (cf. les travaux de l'etholinguiste Boris CYRULNIK, notamment Les nourritures affectives, Paris, Odile Jacob, 1983). Le préfixe « juri » vient directement du latin jus, juris, auquel on doit la famille de termes que sont juridiction, juridique, jurisprudence, juriste (cf. Alain REY, 1998, p. 1937 et 1938), etc. et qualifie le courant de la linguistique que des spécialistes - comme le terminologue, le traducteur, le (co)rédacteur, le lexicographe, le réviseur, l'interprète (oral ou de la loi), etc. - appliquent au droit, à son langage et à ses textes.

2. Le « mot », en fait (cf. latin classique verbum, verbi : mot, terme). Le vocable « verbe » ne s'est imposé dans les traductions de la Bible qu'au XVII' siècle, selon A. REY (1998: 4029). 
3. Selon Gérard CORNU (Préface du Vocabulaire juridique , $4^{\mathrm{e}}$ éd., " Quadrige », 2003, p. viii), en formulant l'hypothèse que «le langage du droit présente des marques linguistiques suffisantes pour constituer un langage spécialisé [qui invite à la comparaison] avec d'autres langages techniques», car le langage du droit peut aussi être qualifié de « technique », au sens où l'entendent les dictionnaires (cf. Le Petit Robert, V TECHNIQUE, adj. I.1). Voir aussi : Anne WAGNER (2002 : 117).

4. Terme consacré par l'usage (SOURIOUX et LERAT 1975), bien qu'il existe d'autres façons d'envisager la question. Voir, entre autres, la position de V.Z. ZIEMBINSKI ( Le langage du droit et la langue juridique; les critères de leur discernement », in Le langage du droit, Archives de philosophie du Droit, t. 19, 1974, p. 26) qui distingue « langage du droit » (celui dans lequel sont formulées les lois) et « langue juridique » (celle dans laquelle les juristes parlent du droit).

5. Pour une bonne esquisse de l'histoire récente des faits et situations qui ont contribué, par le canal de la lisibilité et du concept anglo-américain de plain language, à donner naissance à la notion actuelle de « jurilinguistique », voir, à l'adresse : www.grandsorganismes.gouv.qc.ca/esimple/activite_2.html.

6. Les actes d'un colloque international tenu à Genève sur la traduction juridique en février 2000 en témoignent, où l'on constate que treize grandes langues - de l'allemand au mandarin en passant par l'espagnol, le grec, le russe ou le portugais - posent, lorsqu'elles sont appliquées au langage du droit, plus ou moins les mêmes problèmes, et que leurs textes présentent des difficultés plus ou moins analogues, compte tenu des pratiques, contraintes et traditions propres à chacune d'elles. Voir : La traduction juridique. Histoire, théorie(s) et pratique. Actes, Bern et Genève, ASTTI / ETI, 2000 [http://www.tradulex.org]

7. Dont Aurel DAVID, pour qui le droit en serait encore à la « pensée naïve » (1982:33); ou le philosophe du droit Michel VILLEY (1975: 6) qui conteste l'existence même d'un langage scientifique du droit. Et jusqu'à un juriste comme F. Karl von SAVIGNY (XIX ${ }^{\text {e }}$.) qui pensait que la langue allemande n'était pas encore prête pour la codification (cf. Sacco, 1999 : 182, note 9 ; Malaurie, 1996: 157).

8. Qui n'hésitent pas à mettre leur expertise au service du public, comme le montrent les nombreux portails, adresses électroniques et sites qui y sont consacrés dans internet. Voir, par ex. : www.laportedudroit.com; www.jurisint.org; www.law-links.ch; www.plainlanguagenetwork.org; http://www.tradulex.org/index.html; www.lisibilite.net; etc. 9. Comme l'a souligné Jean-Paul VINAY, à propos des traducteurs canadiens dont le « rôle dépasse (...) de très loin celui du traducteur européen ou américain (...) au Canada, ils sont responsables de l'évolution de la langue » $(L a$ traduction, une profession, Montréal, Conseil des traducteurs et interprètes du Canada, 1978, p. 22).

10. En témoigne, par ex., le thème du $X^{e}$ congrès international de droit comparé (Bristol, R.-U., 1998,) qui portait sur « Langue et droit».

11. Alors procureur général de la Cour de Cassation, dans une entrevue accordée au Nouvel Observateur, $\mathrm{N}^{\circ} 1932$, 15-21 nov. 2001, p. 56.

12. Source : http://thomas.loc.gov/home/resume/resume/html/

13. Voir en particulier sur ce sujet : http//www.grandsorganismes.gouv.qc.ca.

14. Comme le montre, parmi d'autres, le site de l'Université de Villetaneuse (Paris XIII), où l'on trouvera le «Quadrirédacteur, dictionnaire multilingue juridique et autour», dictionnaire quadrilingue (fr-al-an-it) dont le professeur Pierre LERAT a dirigé la préparation: http://www-lli.univparis13.fr/ressources/quadri/index.php?lang=fr. Voir aussi le Dictionnaire comparé du droit d'auteur et du copyright (de Marie CORNU, Isabelle de LAMBERTERIE, Pierre SIRINELLI et Catherine WALLAERT, Paris, CNRS, 2003) à l'adresse : http://www.bibsciences.org/more.php?rub=cnrs_droit_auteur.

15. Par ex., le Centre de recherche en droit privé et comparé du Québec (CRDPCQ), à Montréal ; le Centre de traduction et terminologie juridiques (CTTJ), à Moncton.

16. L'Institut Joseph-Dubuc du Manitoba et l'ISAIDAT de R. SACCO (Turin), par ex.

17. Tels ceux, nombreux, du CNRS, en France, par ex.

18. Est particulièrement significatif à cet égard le cas du Mexique, stimulé par l'Accord tripartite de libre-échange nord-américain (ALENA/NAFTA) conclu entre le Canada, les É.-U. et lui-même.

19. Je pense en particulier aux stages de formation et de perfectionnement qu'offrent les différents services de ces institutions et organismes, notamment à des traducteurs, terminologues, juristes, juristes-linguistes, etc.

20. Voir le bilan de vingt ans de corédaction des lois dressé par André LABELLE (2000:269).

21. L.R.Q., c. P-40.1.

22. Voir, entre autres, l'Association des usagers de la langue française (ASULF) qui, au Québec, œuvre pour encourager la qualité de la langue. 
23. Non seulement dans l'hémisphère nord, mais aussi dans celui du sud. Par ex.: Argentine, Brésil, Mexique, Vanuatu, ...

24. En lexicographie juridique, par ex., voir le travail d'Ethel GROFFIER et David REED (1990) ; en traduction juridique, celui de Susan SARCEVIC (1997); en linguistique juridique, Gérard CORNU (2000); etc.

25. A qui l'on doit, entre autres publications, The Elements of Legal Style, Oxford / New York, Oxford University

Press, 1991.

\section{RÉFÉRENCES}

Alland, D. et Rials S. (2003) : Dictionnaire de la culture juridique, Paris, PUF.

BADINTER, R. (1991) : «Vive l'écrit », in Le Point 1000, 16 novembre 1991.

CARbonnier, J. (1995) : Flexible droit, $8^{\mathrm{e}}$ éd., Paris, L.G.D.G.

Chodkiewick, C. (2005) : «La description de la langue du droit au moyen des classes d'objets », in $L a$

jurilinguistique : entre langues et droits, GEMAR J.-C. et KASIRER N. (dir.), Montréal, Thémis, p. 23-42.

Cote, P.-A. (1999) : Interprétation des lois, $3^{e}$ éd., Montréal, Thémis.

Cornu, G. (2000) : Linguistique juridique, $2^{\mathrm{e}}$ éd., Paris, Montchrestien.

CORNU, G. (1987) : Vocabulaire juridique, Paris, PUF.

CRDPCQ (1985) : Centre de recherche en droit privé et comparé du Québec, Dictionnaire de droit privé du Québec, Montréal, Université McGill, Faculté de droit.

DARBELNET, J. (1982) : « Niveaux et réalisations du discours juridique », in Langage du droit et traduction,

GEMAR J.-C. (dir.), Montréal, Conseil de la langue française, p. 51-60.

DAVIS, A. (1982) : «Les termes élémentaires du droit », in Langage du droit et traduction, GEMAR, J.-C. (dir.),

Montréal, Conseil de la langue française, p. 31-38.

DoIRON, D. et SNOW G. (2005) : «Bibliographie de la jurilinguistique franco-canadienne », in La jurilinguistique : entre langues et droits, GEMAR J.-C. et KASIRER N. (dir.), Montréal, Thémis, p. 145-160.

DuMEzIL, G. (1995) : Mythe et épopée I. II. III., Paris, Gallimard, "Quarto ».

GALlAS, T. (2002) : « Politique de transparence et accès du public aux documents : une interprétation plus facile du droit communautaire? », in L'interprétation des textes juridiques rédigés dans plus d'une langue, SACCO, R. (dir.), Torino, L'Harmattan Italia, p. 89-102.

GEMAR, J.-C. et KASIRER, N. (dir.) (2005) : La jurilinguistique : entre langues et droits. Jurilinguistics : Between Law and Langage, Montréal, Thémis.

GEMAR, J.-C. (2001) : «Traduire le texte pragmatique. Texte juridique, culture et traduction », in Le facteur culturel dans la traduction des textes pragmatiques, Les Cahiers de l'ILCEA 11, Grenoble, Université Stendhal, p. 11-38.

Gemar, J.-C. (1994) : Fonctions de la traduction juridique en milieu bilingue. Le cas du Canada, thèse de doctorat d'État ès Lettres (Université de Toulouse-Le Mirail) [reprographiée].

GEMAR, J.-C. (1983): Les trois états de la politique linguistique du Québec, Québec, Conseil de la langue française.

GEMAR, J.-C. (dir.) (1982) : Langage du droit et traduction. Essais de jurilinguistique, Montréal, Conseil de la langue française.

GENY, F. (1921) : Science et technique en droit privé positif, Paris, Sirey.

GozZI, P. : «Translation of the Acquis communautaire », in Legal Translation. Preparation for Accession to the European Union, SARCEVIC S. (dir.), Rijeka, Faculty of Law, p. 23-34.

GREENSTEIN, R. (dir.) (2003) : Langue, culture et code : regards croisés, Paris, CERLAC, L'Harmattan.

GROFFIER et REED, D. (1990): La lexicographie juridique, Cowansville (Qué.), Yvon Blais.

LAdMiRal, J.-R. (1988) : «Epistémologie de la traduction », in Textlinguistik und Fachsprache, ARnTZ R. (dir.), Hildesheim, Georg Olms Verlag, p. 35-49.

LABELlE, A. (2000) : «La corédaction des lois fédérales au Canada. Vingt ans après : quelques réflexions », in $L a$ traduction juridique. Histoire, théorie(s) et pratique. Actes, Genève/Bern, ASTTI/ETI., p. 269-284.

LERAT, P. (1995) : Les langues spécialisées, Paris, PUF.

Leroi-Gourhan, A. (1983) : Le fil du temps, Paris, Fayard.

Malaurie, P. (1996) : Anthologie de la pensée juridique, Paris, Cujas.

MELlinKoff, D. (1963): The Language of the Law, Boston, Little, Brown. 
MORIN, E. (1999) : Les sept savoirs nécessaires à l'éducation du futur, Paris, Unesco [http :www.agora21.org/unesco//savoirs].

MoRIN, E. (1984): «L'inséparabilité des notions d'ordre et de désordre », in Ordre et désordre, Neuchatel, Editions de la Baconnière, 1984.

Mounin, G. (1979) : « La linguistique comme science auxiliaire dans les disciplines juridiques », in La traduction juridique, GEMAR, J.-C. (dir.), Meta 24-1, p. 9-17.

NiDA, E. A. (1982) : « Translating Legal Terms in the Bible », in Langage du droit et traduction, GEMAR, J.-C. (dir.), Québec, Linguatech et Conseil de la langue française, p. 261-269.

REY, A. [dir.] (1998) : Dictionnaire historique de la langue française, Paris, Dictionnaires Le Robert (éd. petit format).

Ricoeur, P. (2004) : Sur la traduction, Paris, Bayard.

Rouland, N. (1991) : Aux confins du droit, Paris, Odile Jacob.

SACCO, R. (1999) : «Langue et droit » in Les multiples langues du droit européen uniforme, SACCO, R. (dir.),

Torino, ISAIDAT, L'Harmattan Italia, p. 163-185.

SARCEVIC, S. (1997) : New Approach to Legal Translation, The Hague, Kluwer.

SouriouX, J.-L. et LerAT, P. (1975) : Le langage du droit, Paris, PUF.

VANDERLINDEN, J. (2002) : « Exercice comparatif au départ d'un sujet convenu. Le droit sud-africain entre principe et réalisme », in L'interprétation des textes juridiques dans plus d'une langue, SACCO, R. (dir.), Turin, ISAIDAT, p. 295-319.

Villey, M. (1975) : Philosophie du droit, Paris, Dalloz.

WAgner, A. (2002) «Le langage de spécialité, langage de technicien », in La langue de la Common Law, Paris, L'Harmattan, p. 117-151. 